\title{
Absolute Change From Nadir in Sum of Diameter
}

National Cancer Institute

\section{Source}

National Cancer Institute. Absolute Change From Nadir in Sum of Diameter. NCI

Thesaurus. Code C112218.

The most recently recorded sum of diameters minus the lowest sum of diameters previously recorded. 